\title{
COMPARAÇÃO DE DIFERENTES ABORDAGENS NA MODELAGEM DA DISTRIBUIÇÃO DIAMÉTRICA
}

\author{
Thelma Shirlen Soares ${ }^{1}$, Hélio Garcia Leite ${ }^{2}$, Carlos Pedro Boechat Soares ${ }^{2}$, Antonio Bartolomeu do Vale ${ }^{2}$ \\ ${ }^{1}$ Eng $^{\mathrm{a}}$ Florestal, Dr ${ }^{\mathrm{a}}$., Curso de Engenharia Florestal, UFG, Campus Jataí, Jataí, GO, Brasil - thelma.soares@ hotmail.com \\ ${ }^{2}$ Eng. Florestal, Dr., Depto. de Engenharia Florestal, UFV, Viçosa, MG, Brasil - hgleite@ufv.br; csoares@ufv.br; abvale@yahoo.com.br
}

Recebido para publicação: 10/02/2009 - Aceito para publicação: 10/03/2010

\begin{abstract}
Resumo
O objetivo deste estudo foi avaliar a eficiência da função de distribuição de probabilidade Weibull truncada à direita em relação ao procedimento de passo invariante baseado na relação de percentis da distribuição diamétrica. Verificou-se que o modelo de passo invariante apresenta ajustes e predições mais precisas quando comparado com o procedimento tradicional, sendo mais eficiente.

Palavras-chave: Passo invariante; função Weibull; avaliação de modelo.
\end{abstract}

\begin{abstract}
Comparison of different approaches to diameter distribution modeling. This study evaluated the efficiency of the Weibull probability distribution function truncated to the right in comparison with the step-invariant procedure to characterize the percentiles of the diameter distribution. The results indicated that the step-invariant procedure provides more accurate adjustments and predictions and is more efficient than the traditional procedure.

Keywords: Step invariant; Weibull function; model evaluation.
\end{abstract}

\section{INTRODUÇÃO}

Uma questão fundamental para viabilizar o planejamento e controle da atividade florestal consiste no conhecimento do crescimento e da produção presente e futura de árvores e povoamentos florestais em função da idade, qualidade de sítio e densidade de plantio, principalmente quando as florestas são destinadas ao aproveitamento múltiplo da produção (MAESTRI, 2003). Sendo as florestas sistemas biológicos dinâmicos que estão continuamente mudando, a predição do valor potencial da produção, por meio de modelos de crescimento e produção, sob várias condições fornecerá informações relevantes para auxiliar na tomada de decisão para se efetuar a otimização da produção florestal (VANCLAY, 1994).

De acordo com Rennolls et al. (1985) e Glade (1986), a predição da distribuição diamétrica em um povoamento é de suma importância no manejo florestal, sendo indispensável para produzir o efeito das diferentes práticas de manejo sobre o volume por classes diamétricas, para definir o tipo de manejo que maximize a renda líquida por hectare e também com relação à tendência mundial de exploração florestal mecanizada, a qual requer, para o seu planejamento e justificativa econômica, o conhecimento da distribuição diamétrica da floresta.

As projeções da estrutura diamétrica consideram a provável distribuição que apresentarão os diâmetros em uma idade determinada, empregando modelos de distribuição diamétrica que recuperam os parâmetros de funções densidade de probabilidade (f.d.p.). Dentre as f.d.p., a função Weibull destaca-se no setor florestal pela sua flexibilidade e capacidade de descrever diferentes tendências e pela facilidade de correlação de seus parâmetros com variáveis do povoamento, o que a torna superior às demais. No entanto, conforme relatam Araújo Júnior et al. (2008), um inconveniente dessa função é que as estimativas do número de árvores por classe muitas vezes ultrapassam o diâmetro máximo observado no povoamento, ou seja, ela não estima para um intervalo de diâmetros $\left(d a p_{\min }<d a p<d a p_{\operatorname{máx}}\right)$ o mesmo número de árvores observado para este intervalo considerado. 
Nesse contexto, o aprimoramento dos métodos de predição é essencial para propiciar estimativas compatíveis e consistentes. Estudos para solucionar tal inconveniente demonstram que a utilização truncada da função, ou seja, funções que fazem com que toda a quantidade de árvores observadas para um intervalo sejam estimadas necessariamente para este mesmo intervalo considerado, tem sido uma alternativa viável para aprimorar as estimativas (SILVA, 2001; ARAÚJO JÚNIOR, 2009).

Este estudo teve como objetivo avaliar a eficiência da f.d.p. Weibull truncada à direita e o procedimento de passo invariante na modelagem do crescimento e da produção, testando a hipótese de que o procedimento de passo invariante apresenta melhores ajustes em relação à f.d.p. Weibull truncada à direita.

\section{MATERIAL E MÉTODOS}

\section{Descrição dos dados}

Foram utilizados dados provenientes de povoamentos do híbrido Eucalyptus grandis W. Hill ex Maiden x Eucalyptus urophylla S. T. Blake, implantados no espaçamento de $3 \times 2 \mathrm{~m}$, localizados na região nordeste do estado da Bahia. Os dados utilizados são advindos de 173 parcelas e compreendem plantios com idades entre 25 e 89 meses. Esses dados foram agrupados em classes de $1 \mathrm{~cm}$ de diâmetro, e optou-se por classes de diâmetro com um intervalo para obtenção de uma melhor visão da estrutura da população florestal. O número de classes por parcela variou em função da quantidade de árvores e da diferença entre os diâmetros mínimo e máximo observados em cada unidade amostral. A frequência de indivíduos por classe de diâmetro foi utilizada para o ajuste da f.d.p. Weibull.

\section{Análises}

$\mathrm{Na}$ área florestal, os modelos Weibull mais utilizados são o de dois e três parâmetros. Porém, considerando as afirmações de Maltamo et al. (1995) de que a função Weibull de dois parâmetros é mais segura e dá maior liberdade ao parâmetro de forma, tornando-a ainda mais flexível para descrever diversas tendências, optou-se pelo uso da função de dois parâmetros.

A f.d.p. Weibull com dois parâmetros foi empregada para a obtenção da proporção de árvores em cada classe de diâmetro. Entretanto, com o intuito de aprimorar as estimativas, efetuou-se o truncamento da função, cuja relação funcional da f.d.p. e a distribuição Weibull truncada à direita ficaram definidas, respectivamente, por:

$$
\begin{aligned}
& \left.f(x ; \beta, \gamma)=\frac{\beta \gamma x^{\gamma-1} e^{-\beta x}}{1-e^{-\beta T} \gamma} ; \beta>0, \gamma>0,(0<x<T)\right) \\
& F(X)=\frac{1-e^{-\beta x^{\gamma}}}{1-e^{-\beta T^{\gamma}}} ;(0<x<T)
\end{aligned}
$$

Em que: $\beta=$ parâmetro de escala;

$\gamma=$ parâmetro de forma;

$T=$ ponto de truncamento (diâmetro máximo).

Para a estimação dos parâmetros da f.d.p. foi empregado o método da máxima verossimilhança, sendo utilizada a metodologia proposta por Gove; Fairweather (1989).

\section{Redistribuição teórica dos diâmetros por classe}

Para avaliar as projeções dos diâmetros por classe diamétrica, efetou-se a redistribuição teórica das frequências por dois diferentes métodos: relacionando parâmetros da função com características do povoamento (empregando-se nesse caso a função Weibull truncada à direita) e o procedimento de passo invariante (cujas projeções são baseadas em percentis).

No primeiro método, a redistribuição teórica dos diâmetros por classe dos parâmetros foi realizada a partir da estimação, por regressão, dos coeficientes $\beta$ e $\gamma$, utilizando variáveis relacionadas 
com os atributos do povoamento, como idade, diâmetro médio, número de árvores, altura dominante, com base no seguinte sistema de equações:

$$
\begin{aligned}
& \ln \hat{H} d=3,5700-25,7843 I^{-1} \\
& \bar{D}=6,620468-0,052552 \mathrm{~N}+0,406683 \mathrm{Hd} \therefore R^{2}=92,4 \% \quad \therefore S_{y x}= \pm 0,83 \mathrm{~cm} \\
& \gamma=1,810831-0,016685 I+0,296597 \bar{D}-0,085201 \mathrm{Hd} \therefore R^{2}=45,8 \% \therefore S_{y x}= \pm 0,63 \\
& \beta=-2,11962+0,004404 \mathrm{I}+0,46773 \bar{D} \therefore R^{2}=94,1 \% \quad S_{y x}= \pm 0,39 \\
& \mathrm{~N}_{2}=\mathrm{N}_{1} \mathrm{e}^{-93,72493\left(\mathrm{I}_{2}{ }^{0,000522}-\mathrm{I}_{1}{ }^{0,000522}\right) \therefore R^{2}=92,3 \% \therefore S_{y x}= \pm 0,45}
\end{aligned}
$$

Em que: $H d=$ alturas das árvores dominantes $(\mathrm{m})$;

$I=$ idade (meses);

$\bar{D}=$ diâmetro médio na idade atual $(\mathrm{cm})$;

$\gamma=$ parâmetro de forma da função Weibull;

$\beta=$ parâmetro de escala da função Weibull;

$N_{l}=$ número de árvores por hectare, na idade atual $\mathrm{I}_{1}$;

$N_{2}=$ número de árvores por hectare, na idade futura $\mathrm{I}_{2}$;

$\ln =$ logaritmo neperiano.

Já no procedimento denominado de passo invariante, as medidas de posição (percentis), efetuadas na distribuição em determinada idade, constituem a base da estimativa das magnitudes dessas mesmas medidas em outras idades.

Considerando que apenas dois parâmetros são desconhecidos na função Weibull ( $\beta$ e $\gamma$ ) e baseando-se na metodologia proposta por Guimarães (1994), foram utilizados os percentis correspondentes a $50 \%$ e $75 \%$ do valor assintótica $\left(\mathrm{P}_{50}\right.$ e $\left.\mathrm{P}_{75}\right)$. Então, a partir dos valores de percentis foram estimados os percentis em idades futuras, utilizando-se as equações:

$$
\begin{aligned}
& \mathrm{P}_{\mathrm{f}} 50=\left(1-\mathrm{e}^{-30,36807 \mathrm{I}_{\mathrm{f}}}{ }^{-0,72565} \mathrm{~Pa}_{50}{ }^{-1,26441}\right) \cdot \mathrm{P}_{\mathrm{a}_{50}} /\left(1-\mathrm{e}^{-30,36807 \mathrm{I}_{\mathrm{a}}}{ }^{-0,72565} \mathrm{~Pa}_{50}{ }^{-1,26441}\right) \\
& \mathrm{R}^{2}=82,4 \% \quad \therefore \mathrm{CV}=67,89 \% \\
& \mathrm{P}_{\mathrm{f}} 75=\left(1-\mathrm{e}^{-4,11011 \mathrm{I}_{\mathrm{f}}}{ }^{-0,25093} \mathrm{~Pa}_{50}{ }^{-0,23489}\right) \cdot \mathrm{P}_{50} /\left(1-\mathrm{e}^{4,11011 \mathrm{I}_{\mathrm{a}}{ }^{-0,25093} \mathrm{~Pa}_{50}{ }^{-0,23489}}\right) \\
& \mathrm{R}^{2}=81,3 \% \therefore \mathrm{CV}=66,07 \%
\end{aligned}
$$

Em que: $P_{f}=$ percentil na idade futura;

$P_{a}=$ percentil na idade atual;

$I_{a}=$ idade atual de medição;

$I_{f}=$ idade futura.

De posse dos parâmetros das equações, obtiveram-se, mediante regressão linear simples, as estimativas dos parâmetros da função Weibull truncada e, então, efetuou-se a reconstituição das mesmas.

A partir dos parâmetros estimados, procedeu-se à redistribuição teórica simulada dos diâmetros. $\mathrm{O}$ teste Qui-quadrado $\left(\chi^{2}\right)$, em nível de $1 \%$ de probabilidade, foi aplicado para avaliar a compatibilidade entre a distribuição diamétrica projetada do início para o final de um período de tempo projetado ano a ano.

\section{Predição da produção}

Estimativas de volumes por classe de diâmetro foram obtidas a partir das redistribuições em combinação com a equação de múltiplos volumes:

$$
\begin{gathered}
V=0,00008 d a p^{1,53553} \cdot H t^{1,44819} \cdot\left[e^{-2,25669 T x / d a p)}\right] \cdot\left[1-\left(\text { d.dap }^{-1}\right)^{1-0,00077 . d}\right] \\
\mathrm{R}^{2}=99,9 \% \quad \therefore \mathrm{S}_{y x}= \pm 0,0357 \mathrm{~m}^{3}
\end{gathered}
$$


Em que: $H t=$ altura total $(\mathrm{m})$;

dap = diâmetro com casca medido a 1,3 $\mathrm{m}$ de altura do solo $(\mathrm{cm})$;

$H d=$ altura dominante $(\mathrm{m})$;

$\ln =$ logaritmo neperiano;

dap = diâmetro medido a $1,3 \mathrm{~m}$ de altura do solo $(\mathrm{cm})$;

$d=$ diâmetro comercial $(\mathrm{cm})$;

$V=$ volume (m3);

$e=$ base dos logaritmos neperianos;

$T x$ = variável binária $(0$ para volume ou diâmetro d com casca e 1 para volume ou diâmetro $\mathrm{d}$

sem casca).

Visando verificar a precisão das estimativas obtidas e a ocorrência ou não de tendenciosidade nas mesmas, foi utilizado o teste L\&O, conforme citado por Leite; Oliveira (2002).

\section{RESULTADOS E DISCUSSÃO}

\section{Simulação das distribuições diamétricas}

Após ajustar a f.d.p. Weibull para cada parcela e em todas as idades, elas foram comparadas com os valores observados e testadas pelo teste Qui-quadrado $(\alpha=5 \%)$. Os resultados desse teste foram nãosignificativos para a maioria das medições e remedições das parcelas, rejeitando-se, assim, a hipótese de nulidade não foi rejeitada ( $>>0,05)$, sendo aceito que os diâmetros observados seguem a distribuição Weibull.

O ajuste obtido pela f.d.p. Weibull usando dados de duas parcelas selecionadas ocasionalmente pode ser observado na figura 1 .
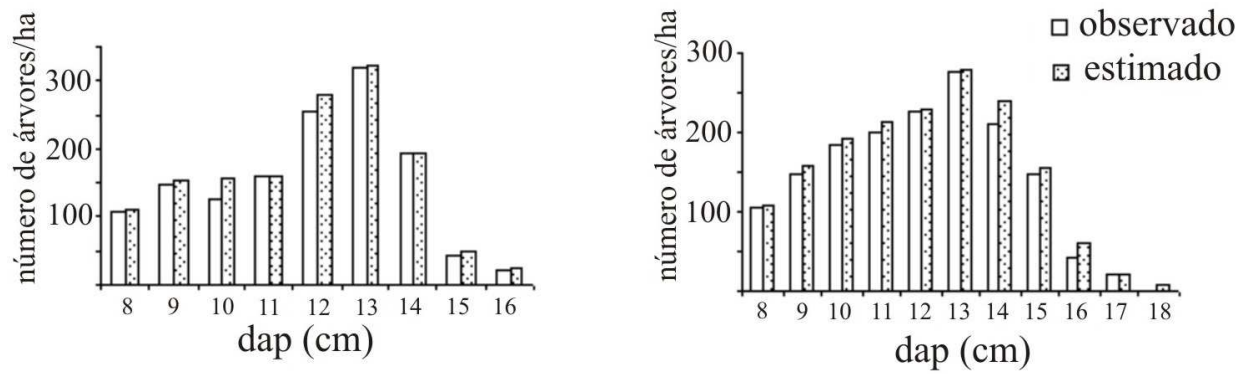

Figura 1. Distribuição de diâmetro, observada e ajustada pela f.d.p. Weibull referente a duas parcelas do povoamento.

Figure 1. Diametric distribution, observed and estimated, for p.d.f. Weibull the two parcels of stands.

O teste de Kolmogorov-Smirnov apresentou resultados não-significativos para todas as parcelas em todas as idades, demonstrando que os diâmetros observados seguem a distribuição Weibull a 5\% de probabilidade.

\section{Redistribuição teórica dos diâmetros e predição volumétrica}

O resultado do teste Qui-quadrado aplicado para avaliar as redistribuições diamétricas realizadas utilizando-se os parâmetros da função com características do povoamento (procedimento A) e o procedimento de passo invariante (procedimento B) é apresentado na tabela 1.

Os resultados do teste Qui-quadrado indicaram predomínio de redistribuições não-significativas, ou seja, para a maioria dos casos houve inexistência de diferenças entre as projeções observadas e estimadas. Verificou-se que a melhor combinação entre f.d.p. e MDD foi a que associou a função Weibull ao procedimento de passo invariante (teste não-significativo em 97,3\% das parcelas avaliadas), a qual foi ligeiramente superior ao procedimento A (utilizando-se os parâmetros da função com características do povoamento). 
Tabela 1. Resultado do teste Qui-quadrado $\left(\chi^{2}\right)$, aplicado para analisar as redistribuições teóricas dos diâmetros.

Table 1. Qui-square test $\left(\chi^{2}\right)$, applied to analyze the theoretical diameters redistributions.

\begin{tabular}{lcc}
\hline \multirow{2}{*}{ Procedimento } & \multicolumn{2}{c}{$\chi^{\mathbf{2}}$} \\
\cline { 2 - 3 } & $* *$ & $\mathbf{n s}$ \\
\hline $\mathrm{A}$ & $2,9 \%$ & $97,1 \%$ \\
$\mathrm{~B}$ & $2,7 \%$ & $97,3 \%$ \\
\hline$* *$ significativo; ns: não-significativo $(\mathrm{p}<0,01)$. &
\end{tabular}

Um exemplo da tabela de produção baseada na distribuição diamétrica projetada empregando os procedimentos A e B é dado na tabela 2 , onde são apresentadas as estimações para algumas parcelas escolhidas ao acaso em diferentes idades, tendo sido estimados o número de árvores sobreviventes, o diâmetro médio, a altura total e o volume.

A variação dos volumes totais do procedimento $b$ em relação ao procedimento $A$ foi de $-4,0 \%$ a $5,5 \%$, sendo que a variação foi maior para os volumes por classe diamétrica do que para os volumes totais em cada idade de projeção.

Para a comparação da similaridade entre as projeções volumétricas obtidas pelo emprego da distribuição pelos diferentes métodos, utilizou-se o teste de $\mathrm{L} \& \mathrm{O}$, cujo resultado indicou não haver diferenças significativas entre os valores observados e ajustados em ambos os casos (Tabela 3).

Verifica-se que ambos os procedimentos propiciam resultados satisfatórios, sem existência de 'bias', comprovando, portanto, a eficiência dos procedimentos utilizados.

Tabela 2. Produção volumétrica baseada nos ajustes da função pelos procedimentos $\mathrm{A}$ e B, em diferentes idades.

Table 2. Volumetric production based on function adjustments by the procedures and A and B, in different ages.

\begin{tabular}{|c|c|c|c|c|c|c|c|}
\hline $\begin{array}{l}\text { Idade } \\
\text { (meses) }\end{array}$ & $\begin{array}{c}\text { Classe } \\
\text { diamétrica } \\
(\mathrm{cm})\end{array}$ & $\begin{array}{l}\text { Número de } \\
\text { árvores } \\
(\mathbf{n} / \mathbf{h a})\end{array}$ & $\begin{array}{l}\text { dap } \\
\text { médio } \\
\text { (cm) }\end{array}$ & $\begin{array}{l}\text { HT } \\
\text { média } \\
(\mathbf{m})\end{array}$ & $\begin{array}{l}\text { VT }\left(\mathbf{m}^{3} / \mathrm{ha}\right) \\
\text { (Proced. A) }\end{array}$ & $\begin{array}{l}\text { VT }\left(\mathbf{m}^{3} / \mathbf{h a}\right) \\
\text { (Proced. B) }\end{array}$ & $\begin{array}{c}\text { Diferença* } \\
(\%)\end{array}$ \\
\hline \multirow{7}{*}{30} & 8,5 & 106 & \multirow{6}{*}{10,8} & \multirow{6}{*}{15,8} & 6,8830 & 6,6122 & $-3,9$ \\
\hline & 9,5 & 170 & & & 8,2080 & 8,3673 & 1,9 \\
\hline & 10,5 & 424 & & & 8,8834 & 9,3269 & 5,0 \\
\hline & 11,5 & 318 & & & 9,2925 & 9,5168 & 2,4 \\
\hline & 12,5 & 85 & & & 9,0427 & 9,1120 & 0,8 \\
\hline & 13,5 & 42 & & & 8,4028 & 8,2979 & $-1,2$ \\
\hline & Total & 1145 & - & - & 50,7124 & 51,2330 & 1,0 \\
\hline \multirow{8}{*}{42} & 8,5 & 127 & \multirow{7}{*}{11,9} & \multirow{7}{*}{18,5} & 7,6531 & 7,5009 & $-2,0$ \\
\hline & 9,5 & 170 & & & 9,4271 & 9,4919 & 0,7 \\
\hline & 10,5 & 233 & & & 10,8700 & 10,5805 & $-2,7$ \\
\hline & 11,5 & 297 & & & 10,5573 & 10,8068 & 2,4 \\
\hline & 12,5 & 127 & & & 11,3610 & 11,2054 & $-1,4$ \\
\hline & 13,5 & 85 & & & 9,7766 & 9,3846 & $-4,0$ \\
\hline & 14,5 & 42 & & & 8,0605 & 8,1244 & 0,8 \\
\hline & Total & 1081 & - & - & 67,7056 & 67,0945 & $-0,9$ \\
\hline \multirow{9}{*}{54} & 8,5 & 1124 & \multirow{8}{*}{13,4} & \multirow{8}{*}{20,1} & 10,9150 & 11,0166 & 0,9 \\
\hline & 9,5 & 42 & & & 13,8123 & 14,1790 & 2,7 \\
\hline & 10,5 & 106 & & & 15,3963 & 15,3311 & $-0,4$ \\
\hline & 11,5 & 233 & & & 15,7256 & 15,9079 & 1,2 \\
\hline & 12,5 & 212 & & & 15,0568 & 15,0437 & $-0,1$ \\
\hline & 13,5 & 318 & & & 13,7186 & 13,3798 & $-2,5$ \\
\hline & 14,5 & 148 & & & 11,8763 & 11,8865 & 0,1 \\
\hline & 15,5 & 42 & & & 10,3499 & 10,2744 & $-0,7$ \\
\hline & Total & 1103 & - & - & 106,8507 & 107,0190 & 0,2 \\
\hline
\end{tabular}




\begin{tabular}{|c|c|c|c|c|c|c|c|}
\hline \multirow{10}{*}{66} & 8,5 & 85 & \multirow{9}{*}{14,6} & \multirow{9}{*}{21,9} & 12,7549 & 12,6722 & $-0,6$ \\
\hline & 9,5 & 127 & & & 16,1403 & 16,0596 & $-0,5$ \\
\hline & 10,5 & 85 & & & 18,0576 & 18,1695 & 0,6 \\
\hline & 11,5 & 255 & & & 18,4437 & 18,4259 & $-0,1$ \\
\hline & 12,5 & 233 & & & 17,6594 & 18,0487 & 2,2 \\
\hline & 13,5 & 212 & & & 16,0816 & 16,1635 & 0,5 \\
\hline & 14,5 & 148 & & & 12,6850 & 12,8506 & 1,3 \\
\hline & 15,5 & 64 & & & 11,0547 & 11,0842 & 0,3 \\
\hline & 16,5 & 42 & & & 8,9303 & 9,1155 & 2,1 \\
\hline & Total & 1273 & - & - & 131,8075 & 132,5897 & 0,6 \\
\hline \multirow{12}{*}{78} & 8,5 & 64 & \multirow{11}{*}{13,8} & \multirow{11}{*}{21,0} & 14,7361 & 14,6350 & $-2,0$ \\
\hline & 9,5 & 42 & & & 20,3393 & 20,5632 & 0,7 \\
\hline & 10,5 & 64 & & & 22,6720 & 22,4026 & $-2,7$ \\
\hline & 11,5 & 212 & & & 23,1569 & 22,9567 & 2,4 \\
\hline & 12,5 & 191 & & & 22,1720 & 21,9944 & $-1,4$ \\
\hline & 13,5 & 255 & & & 20,1911 & 19,8846 & $-4,0$ \\
\hline & 14,5 & 148 & & & 17,4973 & 16,9033 & 0,8 \\
\hline & 15,5 & 127 & & & 15,2486 & 15,4592 & $-0,9$ \\
\hline & 16,5 & 42 & & & 12,3181 & 12,3158 & 0,9 \\
\hline & 17,5 & 42 & & & 10,1023 & 10,6532 & 2,7 \\
\hline & 18,5 & 21 & & & 3,9621 & 4,0172 & $-0,4$ \\
\hline & Total & 1209 & - & - & 182,3956 & 181,7852 & 1,2 \\
\hline \multirow{13}{*}{90} & 9,5 & 85 & \multirow{12}{*}{17,7} & \multirow{12}{*}{25,6} & 16,1363 & 16,2058 & $-0,7$ \\
\hline & 10,5 & 106 & & & 20,4194 & 20,2541 & 1,1 \\
\hline & 11,5 & 255 & & & 22,7613 & 22,5751 & $-1,2$ \\
\hline & 12,5 & 170 & & & 23,2481 & 23,5537 & $-0,9$ \\
\hline & 13,5 & 64 & & & 22,2593 & 22,2192 & $-0,8$ \\
\hline & 14,5 & 106 & & & 20,1714 & 19,7707 & $-1,5$ \\
\hline & 15,5 & 170 & & & 17,5166 & 17,0016 & $-3,4$ \\
\hline & 16,5 & 170 & & & 15,2653 & 15,4031 & 1,4 \\
\hline & 17,5 & 42 & & & 12,3317 & 12,4325 & 0,0 \\
\hline & 18,5 & 106 & & & 10,1134 & 10,0686 & 5,5 \\
\hline & 19,5 & 42 & & & 7,5757 & 7,4014 & 1,4 \\
\hline & 20,5 & 21 & & & 6,2970 & 6,3184 & $-0,3$ \\
\hline & Total & 1400 & - & - & 194,0955 & 193,2044 & $-0,7$ \\
\hline
\end{tabular}

* Representa a diferença percentual entre o volume total estimado pelo procedimento A e pelo procedimento B.

Tabela 3. Resultados do teste de L\&O para comparação das distribuições volumétricas dos volumes observados e preditos pelos diferentes métodos $(\mathrm{p}>0,05)$.

Table 3. Results of the test of L\&O for comparison of the volumetric distributions of the observed and estimated volumes for the different methods $(\mathrm{p}>0,05)$.

\begin{tabular}{lcccc}
\hline Procedimento & $\mathbf{F}\left(\mathbf{H}_{\mathbf{0}}\right)$ & $\mathbf{t} \bar{e}$ & $r_{Y_{j} Y_{1}}$ & \multicolumn{1}{c}{ Conclusão } \\
\hline A & $0,048 \mathrm{~ns}$ & $2,259 \mathrm{~ns}$ & $\operatorname{sim}$ & $\begin{array}{l}\text { Valores observados }\left(\mathrm{Y}_{\mathrm{j}}\right) \text { são estatisticamente iguais aos } \\
\text { valores estimados }\left(\mathrm{Y}_{1}\right) \text {, para o nível de significância de 5\%. }\end{array}$ \\
B & $0,057 \mathrm{~ns}$ & $2,181 \mathrm{~ns}$ & $\operatorname{sim}$ & $\begin{array}{l}\text { Valores observados }\left(\mathrm{Y}_{\mathrm{j}}\right) \text { são estatisticamente iguais aos } \\
\text { valores estimados }\left(\mathrm{Y}_{1}\right), \text { para o nível de significância de 5\%. }\end{array}$ \\
\hline
\end{tabular}

Portanto, pode-se afirmar que ambos os métodos podem ser empregados para a modelagem da distribuição diamétrica e projeção da produção volumétrica de povoamentos florestais não desbastados, gerando resultados precisos e consistentes. 


\section{CONCLUSÃO}

- Ambos os procedimentos empregados são eficientes na projeção da distribuição diamétrica e projeção da produção de povoamentos que não sofreram intervenções de desbastes, sendo que o modelo de passo invariante gera resultados ligeiramente superiores ao procedimento que relaciona características do povoamento com a função Weibull truncada à direita.

\section{REFERÊNCIAS}

ARAÚJO JÚNIOR, C. A.; PELLI, E.; CASTRO, R. V. O.; OLIVEIRA, M. L. R.; NOGUEIRA, G. S. Ajuste da função Weibull com truncamento à direita. In: Jornada Científica e Tecnológica da UFVJM, X, 2008, Diamantina. Anais... Diamantina: UFVJM, 2008. 1 CD-ROM.

ARAÚJO JÚNIOR, C. A. Projeção da distribuição diamétrica de povoamentos florestais utilizando diferentes formas da função Weibull. 2009. 50 f. Monografia (Graduação em Engenharia Florestal) Universidade Federal dos Vales do Jequitinhonha e Mucuri, Diamantina, MG.

GLADE, J. E. Prognose de volume por classe diamétrica para Eucalyptus grandis Hill Ex Maiden. 1986. 99 f. Tese (Mestrado em Engenharia Florestal) - Universidade Federal do Paraná, Curitiba/PR.

GUIMARÃES, D. P. Desenvolvimento de um modelo de distribuição diamétrica de passo invariante para prognose e projeção da estrutura de povoamentos de eucalipto. 1994. $160 \mathrm{f}$. Tese (Doutorado em Ciência Florestal) - Universidade Federal de Viçosa, Viçosa/MG.

LEITE, H. G.; OLIVEIRA, F. H. T. Statistical procedure to test the identity of analytical methods. Communications in soil science plant analysis, v. 33, n. 7/8, p. 1105-1118, 2002.

MAESTRI, R. Modelos de crescimento e produção para povoamentos de Eucalyptus grandis considerando variáveis ambientais. Curitiba: 2003. 143 f. Tese (Doutorado em Engenharia Florestal) Universidade Federal do Paraná.

MALTAMO, M.; PUUMALINEN, J.; PÄIVINEN, R. Comparison of Beta and Weibull functions for modelling basal area diameter in stands of Pinus sylvestris and Picea abies. Scandinavian Journal of Forest Research, v. 10, p. 284-295, 1995.

RENNOLLS, K.; GEARY, D. N.; ROLLINSON; T. J. D. Characterizing diameter distributions by the use of the Weibull distribution. Forestry, v. 58, n. 11, p. 57-66, 1985.

SILVA, A. A. L. Emprego de modelos de crescimento e produção em análise econômica de decisões de manejo florestal. 2001. 79 f. Dissertação (Doutorado em Ciência Florestal) - Universidade Federal de Viçosa, Viçosa, MG.

VANCLAY, J. K. Modeling forest growth and yield: applications to mixed tropical forests. Copenhagen: CAB International, 1994. 312 p. 
FLORESTA, Curitiba, PR, v. 40, n. 4, p. 731-738, out./dez. 2010 Soares, T. S. et al. 